\title{
Slurry Pipeline for fluid transients in pressurized conduits
}

\author{
Tarik Chakkour and Fayssal Benkhaldoun
}

\begin{abstract}
Morocco is known by the pipeline from Khouribga to Jorf Lasfar that is considered as one of the most world's largest for the slurry transportation. This phosphate slurry undergoes different manufacturing process. During this process, the rheological properties of the slurry have been taken into account, and next adapted for our study. There are numerous approaches in the literature which investigate different Eulerian-Lagrangian, Eulerian-Eulerian and Stochastic models to simulte the slurry flow [1], [2]-[3]. Actually, it is very difficult to consider all variables for establishing a general model, we build an Eulerian and a homogeneous one in easier framework. Among these variables, there is the stress tensor which is involved in the model. Since it is considered null, then the non-Newtonian fluid is approched by multiple friction factors. In the present work, a onedimensional three-fluid model is developed in Python. The physical model features a mass and momentum balance for each fluid. It allows to predict the pressure drop and flow patterns. The hydraulic transport of slurry system in horizontal tubes has been investigated. To simulate it dynamically, continuity and momentum equations used in applied engineering problem, are solved together. These equations are conveniently solved using the method of characteristics (MOC). The reason for utilizing this method is the robustness and efficiency compared to the finite volume method (FVM). The originality for this work takes into account the physical discontinuity at interface separating slurry and water which mix with each other. The numerical results from the numerical code model head and pressure losses. We test numerically the fitting of the model with the real physical problem. Then the model is used on simplified examples in order to show its capability to be used to predict the flow behaviour in different regimes, showing consequently its consistency.
\end{abstract}

Keywords - Slurry shield, Pipeline transport, homogeneous singlephase, method of characteristics, non-Newtonian fluids.

\section{INTRODUCTION}

$\mathbf{T}$ ODAY in the world, the phosphate is the commercial source used as raw material for manufacturing phosphoric acid, even in food and pharmaceutical production. The considered fluid is the slurry which contains a variety of materials. In this study, it is a mixture between phosphate particles and water. The slurry flow is always the principal challenge of computational fluid dynamics. There are many reasons for this challenge. In fact, it contributes to innovate the slurry transportation and manufacturing process. Developing its transport allows to preserve the countries water and its ecosystem.

The authors of this paper investigate the hydraulic transport of particles in tubes. First, F. Benkhaldoun built in [4] the mathematical model of water flows and the transport-diffusion

M. Chakkour is with the Department of Mathematics, ENSTA ParisTech, PALAISEAU, France, e-mail: tarik.chakkour@ensta-paris.fr. M. Benkhaldoun is head team of scientific computing, UMR 6205, LAGA, University Paris 13, France

Manuscript received December 4, 2019; revised February 5, 2020. processes of pollutants. In the sequel, T. Chakkour uses in [5] the modeling approaches $(0 \mathrm{D} / 1 \mathrm{D})$ for numerical simulations in tubes with abrupt contraction on OpenFoam (simulation software CFD 3D). Many works focus on multiphase flow models in which the phases are strongly coupled. The main difficulties in these models are due to the interfaces between the phases, and the discontinuities associated to them (Ishii \& Mishima in [6], 1984). The originality of this work is to present in simplified form a homogeneous single-phase model. The most important advantage of this model is the considerably smaller number of variables to be solved compared to the multiphase model. Then, numerical approximations are less made to simulate the transport of slurry utilizing a singlephase model. On other hand, mathematical and experimental heterogeneous model are investigated in [7].

In this paper, a one-dimensional three-fluid model is established based on single-phase flow theory by considering the rheological properties of the fluid. Conceivably, numerical simulation has been widely used to study the characteristics of pipeline transportation and desirable results have been achieved. Subsequently, the transport of slurry over the pipeline is investigated through numerical simulation based on numerical code.

The pipeline was primarily perceived as a means of transporting different materials. In [8], the authors deal with multiple simulation techniques that are used to simulate the operation of the Antamina pipeline system. In the present work, the slurry pipeline [9], [10] is designed to transport phosphate rock from Khouribga's minings to the Jorf Lasfar chemicals plants, this $187 \mathrm{~km}$ long slurry pipeline is scheduled to be complete in 2025 . The pipeline will generate substantial savings in transport cost, as well as in water and energy. We carried out a mathematical modeling on a number of parameters influencing the process of conducting phosphate through the pipeline. In this way, we have been interested in modeling the linear or regular load losses, the hydraulic gradient, the pressure in any point of the pipeline The result is grouped into the numerical code written in Python that can provide us the behavior of the pressure drops, and the piezometric line along the pipeline.

The paper is organized as follows. We start with description of the mathematical model which is presented in detail with the assumptions, see section II. The method of characteristics (MOC) with time marching procedure is employed for finding the solutions. In section III, we study the Darcy friction factor that is solved from the Swamee-Jain equation. Next, we deal in section IV with the numerical discrete method for solving the original continuity and momentum equations. We propose 
in section V physical steady-state solutions for only one fluid. By comparing with explicit solutions, independent-time state occurs, proving the validation of model. We investigate in section VI the unsteady-state for three-fluid model without any specific conditions. While, we treat in section VII the same model with artificial conditions. Finally, the aimed work is recapitulated in the conclusion section VIII.

\section{Method of ChaRACTERISTICS FOR TRANSIENT FlOW}

In this section the differential equations of motion and continuity are developed for liquid flow through a conical tube as well as for a cylindrical tube. In order to present these equations in their simplest form, the terms of lesser importance are omitted from the equations. Denoting by $A$ the cross sectional area that the fluid crosses the tube. The area $A$ is, in general, a function of $x$, which is the coordinate distance along the axis of the tube from the arbitrary origin. The tube is inclined with horizontal at an angle $\theta$, positive when the elevation increases in the positive $x$ direction. The influence of pipe inclination has been studied on mainly works [11], [12]

The continuity and momentum equations form a pair of quasi-linear hyperbolic partial differential equations in terms of two dependant variables, discharge $Q$ and hydraulic-gradeline elevation $H$ sometimes called the piezometric head, or in short, the head. The continuity equation in terms of discharge $Q=V A$ instead of the flow velocity $V$ and piezometric head $H$ leads to:

$$
\frac{\partial H}{\partial t}+\frac{Q}{A} \frac{\partial H}{\partial x}-\frac{Q}{A} \sin (\theta)+\frac{a^{2}}{g A} \frac{\partial Q}{\partial x}=0,
$$

in which $a$ is the acoustic wave speed or a wave celerity of the fluid. The wave speed describes the speed of pressure waves travelling through a fluid medium. The wave speed in a closed conduit is directly determined by the physical properties of conduit and fluid. We present here the wave speed $a$ that is a useful parameter for few special elastic conduits with Poisson's ratio $\mu$ of the material, in which the walls is relatively thick in comparison with diameter. In this condition, the constant $a$ depends on the property of the pipe which is the wall thickness $e$. The wave speed propagation $a$ for transient flow condition in the pipe:

$$
a=\sqrt{\frac{\frac{K}{\rho}}{1+\frac{C K D}{E e}}},
$$

where $K$ and $\rho$ are the bulk modulus of elasticity and mass density of the fluid, $E$ is the Young modulus (modulus of elasticity) of the pipe material, and $C$ is the parameter for the pipe geometry that depends on the Poisson's ratio $\mu$ of the wall material meaning how well the pipe is supported. Here, $D$ is the pipe diameter. Three support situations for a thin pipeline are examined:

1) $C=1-\frac{\mu}{2}$, if pipe is anchored at its upstream end only

2) $C=1-\mu^{2}$, if pipe is anchored throughout against axial movement
3) $C=1$, if pipe is anchored with expansion joints throughout

For a perfectly rigid pipe, $E$ is infinite, and the wave celerity $a$ is simplified to:

$$
a=\sqrt{\frac{K}{\rho}}
$$

with $K$ and $\rho$ defined as in equation (2) which is only true for single phase fluids. It expresses the wave speed for a few special conduits. If it is assumed that a piepeline contains a fluid which consists of a liquid as water, then this expression is used.

For slurry, there are two ideas to express the wave celerity $a$ for a solid-liquid mixture. The first one is that Kao and Wood [13] considered the surge problem, and developed a mathematical model for the surge pressure in terms of hindered particle drag coefficients in the liquid. Here, since longitudinal effects are ignored, $C$ is equal to 1 , i.e. $C=1$. The inverse mass density $\rho$ is defined as the combination of the fluid and solid concentrations of them inverse densities:

$$
\frac{1}{\rho}=\frac{C_{s}}{\rho_{s}}+\frac{C_{f}}{\rho_{f}},
$$

where $s$ and $f$ represent respectively solid and fluid phases, $C_{s}$ and $C_{f}$ are volumetric concentration of solid and fluid respectively. Variables $\rho_{s}$ and $\rho_{f}$ are respectively solid and fluid densities. The elasticity variable $K$ is similarly decomposed as:

$$
\frac{1}{K}=\frac{C_{s}}{K_{s}}+\frac{C_{f}}{K_{f}}
$$

where, $K_{s}$ and $K_{f}$ are the bulk modulus of solid and fluid elasticity, respectively. Substituting expressions (4) and (5) in equality (2), that achieves the following wave celerity expression [13]:

$$
a=\sqrt{\frac{\frac{C_{s}}{\rho_{s}}+\frac{C_{f}}{\rho_{f}}}{\frac{C_{s}}{K_{s}}+\frac{C_{f}}{K_{f}}+\frac{D}{E e}}} .
$$

It is assumed that the total volume can be expressed as the sum of the liquid and the solid volumes. Then, the sum of the volumetric concentration of solid $C_{s}$ and fluid $C_{f}$ is equal to 1. From this, equality (6) yields:

$$
a=\sqrt{\frac{\frac{C_{s}}{\rho_{s}}+\frac{1-C_{s}}{\rho_{f}}}{\frac{C_{s}}{K_{s}}+\frac{1-C_{s}}{K_{f}}+\frac{D}{E e}}} .
$$

Bechteler and Vogel [14] criticised the physical meaningfulness of the density term in equation (7). Instead, they proposed the speed of sound expression below, in line with the choice of Thorley and Hwang [15]: 


$$
a=\sqrt{\frac{\frac{1}{\rho_{s} C_{s}+\left(1-C_{s}\right) \rho_{f}}}{\frac{C_{s}}{K_{s}}+\frac{1-C_{s}}{K_{f}}+\frac{D}{E e}}} .
$$

Here the mixture density $\rho$ in terms of the fluid and solid densities is given by the following relation:

$$
\rho=\rho_{s} C_{s}+\left(1-C_{s}\right) \rho_{f}
$$

For most engineering applications, the convective acceleration term $\frac{Q}{A} \frac{\partial H}{\partial x}$ and the slope term $\frac{Q}{A} \sin (\theta)$, are very small compared to other terms, thus they may be neglected in equation (1) (chaudhry 1987). Hence, this continuity equation (1) is simplified as:

$$
\frac{\partial H}{\partial t}+\frac{a^{2}}{g A} \frac{\partial Q}{\partial x}=0
$$

In equation (10) the distance $x$ and time $t$ are the independent variables while the piezometric head $H$ and discharge $Q$ are the dependent variables and are functions of $x$ and $t$. We will visualize later $H$ and $Q$ with setting the time $t$ and varying the variable space $x$. Similarly as in derivation of the continuity equation (1), the equation of motion can be derived by applying Newton's second law of motion that takes the following form:

$$
\frac{\partial H}{\partial x}+\frac{1}{g A} \frac{\partial Q}{\partial t}+\frac{Q}{g A^{2}} \frac{\partial Q}{\partial x}+\frac{f Q|Q|}{2 g D A^{2}}=0 .
$$

The equation of motion (11) is further simplified by dropping the convective acceleration term $\frac{Q}{g A^{2}} \frac{\partial Q}{\partial x}$ which is very small compared to other terms (chaudhry 1987). Hence, the equation (11) can be written in the following form:

$$
\frac{\partial H}{\partial x}+\frac{1}{g A} \frac{\partial Q}{\partial t}+\frac{f Q|Q|}{2 g D A^{2}}=0 .
$$

Water hammer equations described in [16] form a set of quasi-linear partial differential equations for there is no direct general solution. The standard method for solution of water hammer equations is the method of characteristics (MOC) which is developed by Wylie and Streeter [17]. The transformation by the MOC method of equations (10) and (12) gives four ordinary differential equations on the characteristic lines $C^{+}$and $C^{-}$that are given as follows:

$$
\begin{aligned}
& C^{+}\left\{\begin{array}{l}
\frac{g}{a} \frac{d H}{d t}+\frac{1}{A} \frac{d Q}{d t}+\frac{f Q|Q|}{2 D A^{2}}=0, \\
\frac{d x}{d t}=+a .
\end{array}\right. \\
& C^{-}\left\{\begin{array}{l}
-\frac{g}{a} \frac{d H}{d t}+\frac{1}{A} \frac{d Q}{d t}+\frac{f Q|Q|}{2 D A^{2}}=0, \\
\frac{d x}{d t}=-a .
\end{array}\right.
\end{aligned}
$$

\section{THE FRICTION FACTOR}

In the present work, an effort has been made to give the asymptotic behavior of the quantity that is the friction factor $f$ multiplied by the discharge square $Q^{2}$. This quantity is involved in the last term of motion's equation (11), taking into account the Reynolds number. We assume in this study that the friction factor would be a monotonic function of Reynolds number, leading to the proper result. We look for the proper asymptotic limit friction-discharge term $f Q^{2}$, when the flow is very laminar. This allows to understand how the elevation varies in this case. First, we recall that the Reynolds number Re is a quantity which engineers use to estimate if a fluid flow is laminar or turbulent. Re can be defined as (see [18] for details)

$$
\operatorname{Re}=\frac{\rho Q D}{A \mu} .
$$

In the case of Bingham plastic fluids, another important dimensionless number highlighting the importance of yield stress is the Hedström number He, which is defined as (see [18])

$$
\mathrm{He}=\frac{\rho D^{2} \tau_{0}}{\mu^{2}}
$$

where $\tau_{o}$ is the yield point or yield strength of fluid. The Reynolds number Re expressed in (17) tells if the flow is laminar or turbulent. If the Reynolds number is smaller than the critical Reynolds number $\mathrm{R} e_{c r}$ which is often assumed to be 2300 , i.e $R e_{c r}=2300$, the flow is laminar. After the laminar flow regime follows the transition region. There the flow switches between laminar and turbulent randomly. When the Reynolds number reaches a certain value, the flow turns from transitional to turbulent. The transition region ends approximately at the Reynolds number 4000 . For laminar flow $\mathrm{R} e<\mathrm{R} e_{c r}$, the friction factor $f$ is a function of the Reynolds number (17) only and is given by a Hagen-Poiseuille equation:

$$
f=\frac{64}{\operatorname{Re}} .
$$

Whereas for turbulent flow $\operatorname{Re}>4000$, the friction factor $f$ depends on the average height of roughness projections of pipe wall. The friction factor $f_{w}$ for water can be approximated using the Swamee and Jain's (1976) equation

$$
f_{w}=1.325\left[\ln \left(\frac{\epsilon}{3.7 D}+\frac{5.74}{\operatorname{Re}^{0.9}}\right)\right]^{-2},
$$

in which $\epsilon$ is the average pipe wall roughness height. The equation (20) is used to solve directly for the Darcy-Weisbach friction factor for a full-flowing pipe. It approximates the implicit Colebrook-White equation. From this and according to (17), the friction factor $f_{w}$ can be expressed in term of the discharge $Q$ : 


$$
f_{w}=1.325\left[\ln \left(\frac{\epsilon}{3.7 D}+\frac{\alpha}{Q^{0.9}}\right)\right]^{-2},
$$

in which the quantity $\alpha$ is given by:

$$
\alpha=5.74\left(\frac{A \mu}{\rho L}\right)^{0.9} .
$$

Little works [19], [20] concerning flow structure of nonNewtonian slurry in different flow regimes has been published. The objective of this research [19] is to describe the transport of slurry in a large-scale pipe. The authors investigate in [20] the Bingham plastic rheological equation for non-Newtonian slurry. Under the assumption that the water is air-free, this confirms its Newtonian behaviour because shear rate is constant. Then, the Colebrook-White equation is widely used to compute $f_{w}$ based on its rheological characteristics (detailed below). However, the slurry is categorized as a homogeneous flow, and is non-Newtonian.

Further, since the discharge $Q$ is very small, the friction coeffecient $f_{w}$ given by equality (21) guarantees a null frictiondischarge term $f_{w} Q^{2}$. On the other hand, Darby and Melson [21] obtained an empirical relation which was developed by Darby and Melson [22]. The empirical formula gives the friction component $f_{T}$ in the turbulent flow regime. This component is used for evaluating the Fanning friction factor in the present study, and is given by following equality:

$$
f_{T}=10^{\beta} \operatorname{Re}^{-0.193},
$$

where, parameter $\beta$ is defined as:

$$
\beta=-1.47\left[1+0.146 e^{-2.9 \times 10^{-5} \mathrm{He}}\right] .
$$

In which, Hedstorm number He is expressed by equality (18). An exact description of friction loss for Bingham plastics was fully developed laminar pipe flow by Buckingham [23]. Buckingham-Reiner equation in a dimensionless form reads:

$$
f_{L}=\frac{64}{\operatorname{Re}}\left[1+\left(\frac{\mathrm{He}}{6.2218 \mathrm{Re}}\right)^{0.958}\right] .
$$

Equality (25) expresses the Fanning friction for slurry in laminar regime in terms of the Bingham Reynolds number $\mathrm{Re}$, and the Hedstorm number He, given respectively by (17) and (18). Darby and Melson [21], in view of Churchil [24] and Usagi obtained a single equation for all flow regimes. The modified friction factor $f$ evolved by these authors is the combination of friction factors expressed in laminar and turbulent regimes:

$$
f_{s}=\left[f_{L}^{m}+f_{T}^{m}\right]^{\frac{1}{m}},
$$

where, components $f_{T}$ and $f_{L}$ are given by equations (23) and (25), respectively. And,

$$
m=1.7+\frac{4000 A \mu}{\rho Q D} .
$$

The detailed rheological characteristics for water-slurry are presented to predict the friction factors $f_{w}$ and $f_{s}$. The physical properties are utilized in Figs. 8 and 10, that are measurable, whose values describe a state of a physical system. These values are computed with only pipe inner diameters, i.e. $D=0.9 \mathrm{~m}$. The pipeline was assumed rough showing that the characteristics of the friction factor were different for laminar and turbulent flow. Then, its roughness $\epsilon$ is $2.10^{-5}$. Now, we will explain how the Reynolds number for waterslurry is computed using equation (17). Once its density is determined, the Reynolds number is given. Density can be changed generally by changing either the pressure or the temperature. The effect of pressure and temperature on the densities of water-slurry is small. The density of water-slurry increases at low temperatures. The density $\rho_{w}$ and the dynamic viscosity $\mu_{w}$ of water are given $10^{3} \mathrm{~kg} / \mathrm{m}^{3}$ and $10^{-3} \mathrm{~Pa} . \mathrm{s}$, respectively, at standard temperature $\left(0^{\circ} \mathrm{C}\right)$ and pressure $(1$ atm).

Note that, the slurry is considered as a mixture between very fine particles of phosphate and water. Even this mixture, the slurry behaves like a homogeneous fluid characterized by density $\rho_{s}$ and viscosity $\mu_{s}$. The phosphate dominates the flow with high concentration. In practice, the ratio of volumetric concentration of phosphate is $60 \%$ and of water is $40 \%$. Setting the density of phosphate with $\rho_{p}=2.10^{3} \mathrm{~kg} / \mathrm{m}^{3}$, and using the density $\rho_{w}$ of water, equality (9) gives the mixture density $\rho_{s}=1600 \mathrm{~kg} / \mathrm{m}^{3}$. Considering that the dynamic plastic viscosity of the slurry varies between 0.006 and 0.0102 , and typically limited to simulation the maximum value in simulation, i.e. $\mu_{s}=0.0102$ Pa.s.

Multiplying the friction factor $f_{s}$ defined by equality (26) with the discharge square $Q^{2}$ :

$$
f_{s} Q^{2}=f_{L} Q^{2} \mathrm{e}^{\frac{\ln \left(1+\left(\frac{f_{T}}{f_{L}}\right)^{m}\right)}{m}} .
$$

Substituting expression (17) of the Reynolds number Re into coupled equations (23) and (25), we get:

$$
\frac{f_{T}}{f_{L}}=\frac{10^{\beta}}{64}\left(\frac{\rho D}{A \mu}\right)^{0.807} \frac{Q^{0.807}}{1+\left(\frac{\mathrm{He} A \mu}{6.2218 \rho D}\right)^{0.958} \frac{1}{Q^{0.958}}} .
$$

The equality (29) shows that quantity $\frac{f_{T}}{f_{L}}$ converges to zero when $Q$ goes to zero. If $\frac{f_{T}}{f_{L}}$ tends towards zero, then, using an order 1 limited development of the natural logarithm function ln, we obtain the following expansion of function:

$$
\frac{\ln \left(1+\left(\frac{f_{T}}{f_{L}}\right)^{m}\right)}{m}=\frac{1}{m}+\frac{f_{T}}{f_{L}}+O\left(\left(\frac{f_{T}}{f_{L}}\right)^{2}\right) .
$$

Since the laminar flow friction factor $f_{L}$ is defined by (24), $f_{L} Q^{2}$ is expressed as: 


$$
f_{L} Q^{2}=\frac{64 A \mu Q}{\rho D}\left[1+\left(\frac{\mathrm{He} A \mu}{6.2218 \rho D}\right)^{0.958} \frac{1}{Q^{0.958}}\right],
$$

showing the following equivalence,

$$
f_{L} Q^{2} \simeq \frac{64 \mathrm{He}^{0.958}(A \mu)^{1.958} Q^{0.042}}{(6.2218)^{0.958}(\rho D)^{1.958}} .
$$

From this, and according to equality (28), the expanded expression $f_{s} Q^{2}$ is obtained:

$$
f_{s} Q^{2} \simeq \frac{64 \mathrm{He}^{0.958}(A \mu)^{1.958} Q^{0.042}}{(6.2218)^{0.958}(\rho D)^{1.958}}
$$

\section{FINITE-DIFFERENCE EQUATIONS}

This section deals with the numerical discrete method for solving the original partial differential equations. To proceed with the numerical solution, the pipe length is divided into $N$ sub-intervals of equal length $\Delta x$ as illustrated in Fig. 1. The $x$ domain starts at the pipe entrance $x_{1}=0$ and ends at the exit $x_{N+1}=L$. The variables $H$ and $Q$ are then computed in the discrete domain shown in Fig. 1. Since the physical information are needed from nodes $x_{1}, x_{2}, \ldots, x_{N+1}$ to travel along the characteristic lines, a time-step size $\Delta t$ is computed using following formula:

$$
\Delta t=C_{r} \frac{\Delta x}{a},
$$

where the Courant number $C_{r}$ must be less than or equal to 1 [25]:

$$
C_{r} \leq 1
$$

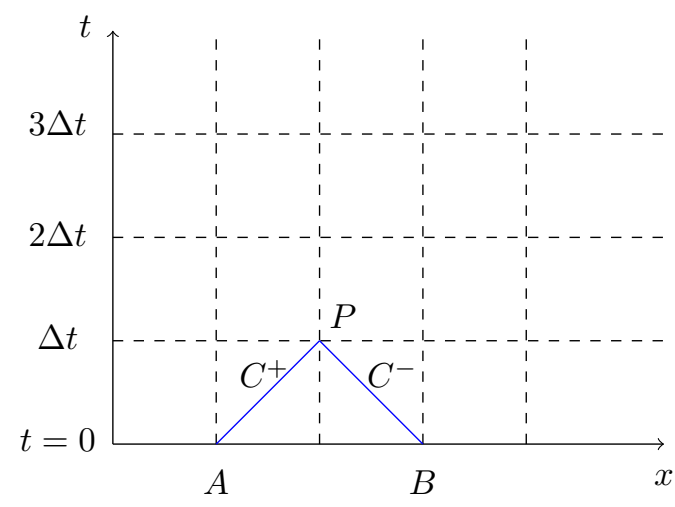

Fig. 1: Numerical characteristics grid lines on the $x t$ plane.

Thus, the size of $\Delta x$ and the wave celerity $a$ (see (2), (3), (6), (7)) determine the size of our time interval. Defining the characteristic lines $C^{+}$and $C^{-}$respectively by lines $A P$ and $B P$, given previously by (13) and (15). Next, assuming that the variables $H$ and $Q$ are known at points $A$ and $B$. The aim here is to know these variables at point $P$. For that, the integration method between these points solves equations (13) and (15) with regard to $H$ and $Q$ :

$$
\begin{gathered}
H_{P_{i}}=\frac{1}{2}\left[\left(H_{i-1}+H_{i+1}\right)+B\left(Q_{i-1}-Q_{i+1}\right)+\right. \\
\left.R_{i+1} Q_{i+1}\left|Q_{i+1}\right|-R_{i-1} Q_{i-1}\left|Q_{i-1}\right|\right] \\
Q_{P_{i}}=\frac{1}{2 B}\left[\left(H_{i-1}-H_{i+1}\right)+B\left(Q_{i-1}+Q_{i+1}\right)\right. \\
\left.-R_{i+1} Q_{i+1}\left|Q_{i+1}\right|-R_{i-1} Q_{i-1}\left|Q_{i-1}\right|\right] .
\end{gathered}
$$

In which, $B$ is the Allievi constant, $R_{A}$ and $R_{B}$ are the resistance coefficients called the radius of gyration defined respectively at points $A$ and $B$, which are given by:

$$
B=\frac{a}{g A}, R_{A}=\frac{f_{A} \Delta x}{2 g D A^{2}}, R_{B}=\frac{f_{B} \Delta x}{2 g D A^{2}} .
$$

The stability analysis takes a huge role in numerical schemes allowing to perform this model problem. Hence, the stable solution should be smaller than a certain critical value. The numerical stability is developed using Von Neumann analysis, satisfying inequality (35). In which, the Courant number $C_{r}$ is given by:

$$
C_{r}=\frac{f \Delta Q \Delta t}{4 D A}
$$

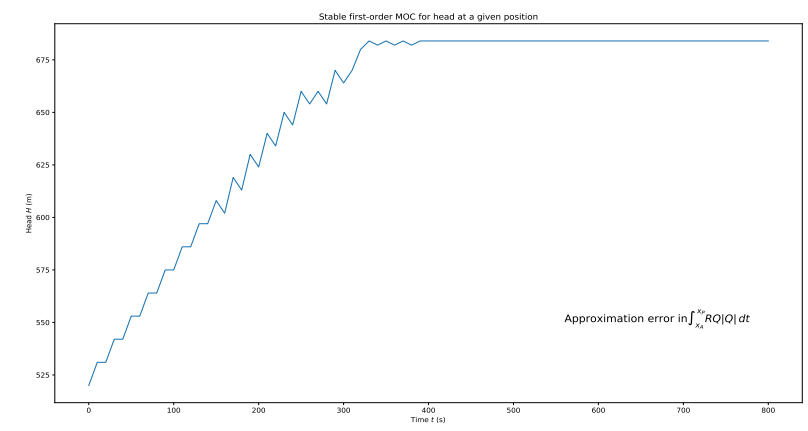

Fig. 2: Stable first-order MOC for head at a given position.

The authors in [26] investigate the numerical stability and accuracy of the MOC method by analyzing typical piping systems with some parameters and empirical stability limits. They studied predictor-corrector procedure. First, predict values for head-discharge are used to obtain secondly a corrected values, limiting consequently time computation. Noticing that if friction loss term is supposed large, then finite-difference scheme is unstable even if inequality (35) is satisfied. They establish the following stability criterion. When the Courant condition $C_{r}$ value is less than 0.5 , the first-order approximation yields stable results. This approximation works well while 
the friction term is small. Further, a second-order method of characteristics is conditionally stable for $C_{r}$ value lower than 0.79 , i.e. $C_{r} \leq .79$. In order to illustrate this stability for $C_{r} \leq 0.5$ using first-order MOC. Fig. 2 reports that head $H$ reaches its peak that its maximum is near to attempt exact value.

\section{STATIONARY SOLUTIONS}

The aim here is to determine the steady fluid flow. We give an explicit analytic representation of the stationary solutions of equations (10) and (12), and compare with the approximated solutions which are illustrated by the numerical simulations. The stationary solution still can be computed explicitly. Finally, we study the goodness of the approximations using the perturbation techniques.

\section{A. Initial and boundary conditions}

Boundary conditions (see Fig. 3) are essentially a relationship between head $H$ and discharge $Q$ at a boundary node, which can then be solved with the characteristic equations to provide a full solution for the boundary. In the models the head-discharge relationship at the boundary can often be solved simultaneously with the appropriate characteristic equations to produce a direct solution. Each boundary condition is solved independently of the other boundary, and independently of the interior point.
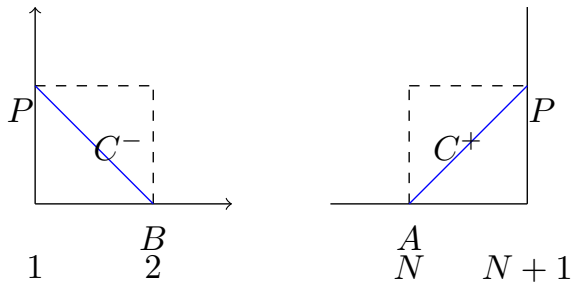

Fig. 3: Characteristics at boundaries.

We use Dirichlet boundary condition to get the stationary solutions. We impose at the upstream and downstream ends the same value of discharge $Q_{0}$ that reads as:

$$
Q\left(x_{N+1}\right)=Q\left(x_{1}\right)=Q_{0} .
$$

We recall that $x_{1}, x_{2}, \ldots, x_{N+1}$ are the nodes of the physical domain. Denoting the upstream boundary head $H\left(t_{1}, x_{1}\right)$ by $H\left(x_{1}\right)$, and the downstream boundary head $H\left(t_{1}, x_{N+1}\right)$ by $H\left(x_{N+1}\right)$. The quantity $H\left(x_{N+1}\right)$ is expressed in terms of $H\left(x_{1}\right)$ :

$$
H\left(x_{N+1}\right)=H\left(x_{1}\right)-\frac{f L Q|Q|}{2 g D A^{2}} .
$$

Initializing the head $H$ with constant $H\left(x_{1}\right)$, meaning that:

$$
\forall i \in \llbracket 1 ; N+1 \rrbracket, H_{0}\left(x_{i}\right)=H\left(x_{1}\right) .
$$

The initial discharge is defined by constant $Q_{0}$ :

$$
\forall i \in \llbracket 1 ; N+1 \rrbracket, Q_{0}\left(x_{i}\right)=Q_{0} .
$$

\section{B. Exact solutions}

Consider a fluid entering a circular pipe presented in Fig. 4. A stationary state of the model is constituted by the conservative variables $H$ and $Q$ that remain in the same state. According to equation (10), if the head $H$ is independent of time, then the discharge $Q$ is constant in space. Otherwise, the discharge $Q$ does not vary over time, the discharge $Q$ is constant in space and time. In the steady-state, the discharge $Q$ is equal to constant $Q_{0}$ :

$$
Q(t, x)=Q_{0}
$$

From this, equation (12) yields:

$$
\frac{\partial H}{\partial x}=-\frac{f Q|Q|}{2 g D A^{2}},
$$

which is coupled with equality (44) to get:

$$
\frac{\partial H}{\partial x}=-\frac{f Q_{0}\left|Q_{0}\right|}{2 g D A^{2}} .
$$

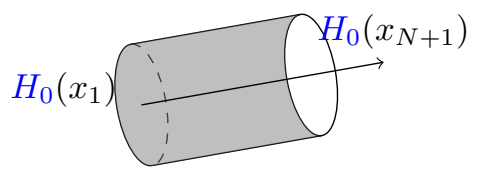

Fig. 4: The flow of a fluid in a circular pipe.

We notice that the friction $f$ depends on the discharge $Q$ which is a constant. By integrating equation (45) between inferior bound $x_{1}$ and superior bound $x$, we get:

$$
H(t, x)=H\left(x_{1}\right)-\frac{f Q_{0}\left|Q_{0}\right|}{2 g D A^{2}}\left(x-x_{1}\right) .
$$

Equation (47) implies that the head $H$ is an affine function of a decreasing slope. It can be given in the discrete form:

$$
\forall i \in \llbracket 2 ; N \rrbracket, H\left(x_{i}\right)=H\left(x_{1}\right)-\frac{f Q_{0}\left|Q_{0}\right|}{2 g D A^{2}}\left(x_{i}-x_{1}\right) .
$$

We present the numerical results in Figs. 5-6 that are shared into five pictures. Fig. 5 shows the evolution of head over space in the stationary state.

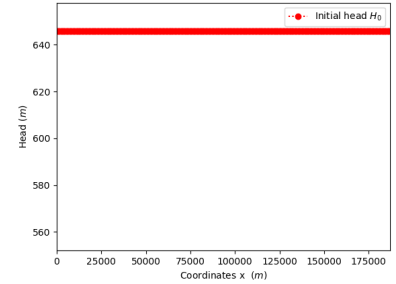

(a) Initial head $H_{0}(x)$

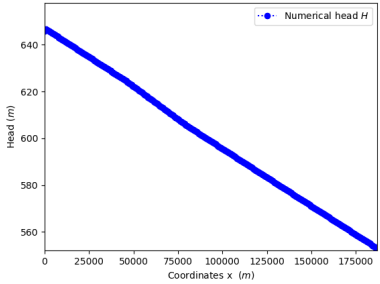

(b) Head $H(t, x)$ at $T_{\max }$.
Fig. 5: Evolution of head over space in steady-state. 
The initial head $H_{0}$ is presented in picture 5a. The head generated by the numerical code using the method of characteristics is the same that is the exact one given by equality (47). It is concluded that these results are consistent with the model. Initializing discharge in 6a with the value $Q_{0}$. At the final stage of time, the numerical discharge $Q$ is shown in picture $6 \mathrm{~b}$. The slant of view of this simulation is interpreted as follows. The discharge $Q$ remains in the same state as time elapses, in every observable instant. This means that it is unchanging in time. Indeed, this discharge $Q$ is equal to the constant $Q_{0}$ (see equality (44)). Now, setting a scale with precision near from $10^{-15}$, the numerical oscillations appear, meaning of numerical noises. This discharge $Q$ is presented in picture 6c. Consequently, the steady-state is approached asymptotically, proving that state variables are unchanging in continuous time.

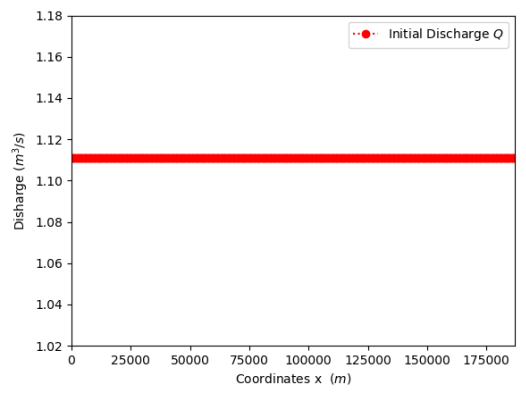

(a) Initial discharge $Q_{0}(x)$.

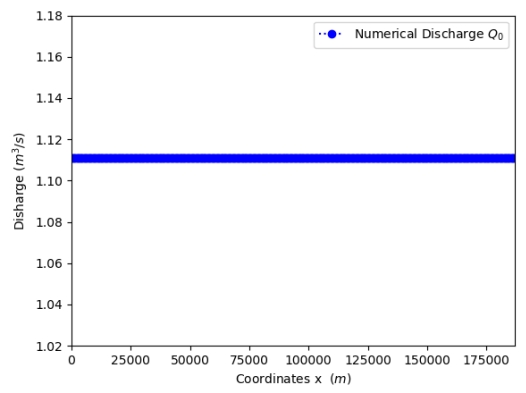

(b) Discharge $Q(t, x)$ at $T_{\max }$.

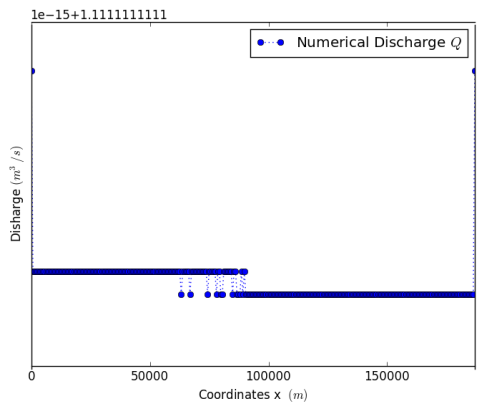

(c) Numerical discharge $Q(t, x)$ at $T_{\max }$ with precision.

Fig. 6: Evolution of discharge over space in steady-state.

\section{INSTATIONARY SOLUTIONS}

This section essentially deals with the so-called three-fluid model. In this model, we focus on the study the instationary flow. The pipeline is divided into three sections as shown in Fig. 7. Consider a water streaming the first section which is defined between two points $x_{1}$ and $x_{N_{1}+1}$. Next, the slurry flows in the second one $\left[x_{N_{1}+1}, x_{N_{2}+1}\right]$. The water dominates the third section $] x_{N_{2}+1}, x_{N+1}$ ] allowing the slurry to exit from the pipeline. We assume that there is no direct contact between water and slurry. The first water domain is subdivided into a finite number $N_{1}$ of cells. These cells cover entirely this computational domain. Similarly, $N_{2}-N_{1}$ means the number of slurry cells that we will give its value later.

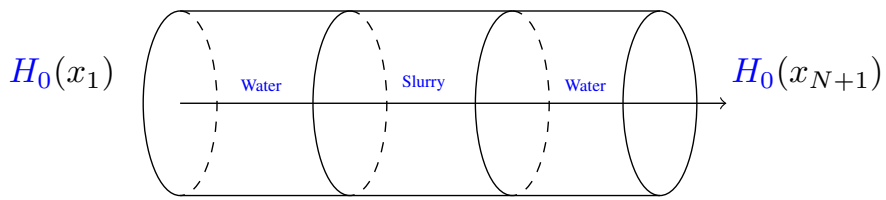

Fig. 7: Schematics of water-slurry-water flow in a circular pipe.

It is necessary to know the initial conditions in order to be able to set the system of equations (10) and (12). Initialise this state involves running the head-discharge over time using equalities (36) and (37). It leads to determine the pressure drop in next discrete step knowing its previous value in time. A shift in the elevation start takes place, meaning that water begins to flow above slurry. The target of this shifted hydraulic head is to cause the unsteady state in the system. We assume in this case a constant rate $Q_{0}$ for the initial discharge $Q_{0}^{i}$ :

$$
Q_{0}^{i}(x)=Q_{0},
$$

and the initial head $H_{0}^{i}$ is obtained by following equality:

$$
H_{0}^{i}(x)=\left\{\begin{array}{l}
H\left(x_{1}\right)-\frac{f_{w} Q_{0}\left|Q_{0}\right|}{2 g D A^{2}}\left(x-x_{1}\right), x \in\left[x_{1}, x_{N_{1}+1}\right], \\
\left.\left.H\left(x_{1}\right)-\frac{f_{s} Q_{0}\left|Q_{0}\right|}{2 g D A^{2}}\left(x-x_{1}\right), x \in\right] x_{N_{1}+1}, x_{N_{2}+1}\right], \\
\left.\left.H\left(x_{1}\right)-\frac{f_{w} Q_{0}\left|Q_{0}\right|}{2 g D A^{2}}\left(x-x_{1}\right), x \in\right] x_{N_{2}+1}, x_{N+1}\right],
\end{array}\right.
$$

in which $f_{w}$ and $f_{s}$ are the friction factors for water and slurry, which are computed according to equalities (21) and (27), respectively. The speeds of sound in water and slurry are given by quantities $a_{w}$ and $a_{s}$, which are computed using equalities (2) and (6), respectively.

The nature of boundary conditions used here is explained as follows. We apply the Dirichlet boundary conditions for the variable $H$. It means that $H\left(x_{1}\right)$ corresponds to the value of head $H$ at the inlet. The relation between the head $H$ at the upstream boundary $H\left(x_{1}\right)$, and at the downstream boundary $H\left(x_{N+1}\right)$ is given by the following equality: 


$$
H\left(x_{N+1}\right)=H\left(x_{1}\right)-\frac{f_{w} L Q_{0}\left|Q_{0}\right|}{2 g D A^{2}} .
$$

For the discharge $Q$, the Neumann condition is imposed at the upstream boundary while the Dirichlet condition is adjusted at the downstream boundary.

In the remainder of this paper, we set the distance 47000 meters of the node $x_{N_{1}+1}$ in where the slurry begins to travel. Setting the position $x_{N_{2}+1}$ at where the interface of slurry ends to value 80000 meters. The discrete number of slurry cells is given by:

$$
N_{2}-N_{1}=\left\lfloor\frac{x_{N_{2}+1}}{\Delta x}\right\rfloor-\left\lfloor\frac{x_{N_{1}+1}}{\Delta x}\right\rfloor .
$$

For our numerical setup, the slurry grid size is $10^{4}$, i.e.

$$
N_{2}-N_{1}=10^{4} .
$$

In what follows, we detail here how the interface slurry-water is solved. There are any restrictions for treating the interface linking the water and slurry, and conversely. For instance, ghost cells are not used to close the computational interfaces. The initial interface water-slurry is physically discontinuous in nature. The value at this interface is defined as the averaged value of left and right limits that is updated at each discrete-time. Consequently, the ended water cell influences the beginning slurry cell which comes after it. Similarly, the last slurry node effects the starting water node.

We know that the experimental results are essential to be compared with the numerical ones. However, these information are purposely censored because, for many confidentiality reasons. The missing data will not affect the fitting of the model because it will be validated numerically with only interpretation. In addition, the properties of the used fluids are shown bellow by table I.

TABLE I: General input datas describing parameters and their values used in model.

\begin{tabular}{ccc}
\hline Physical variables & Numerical values & Dimensions \\
\hline Pipe length, $L$ & $187.10^{3}$ & $\mathrm{~m}$ \\
\hline Diameter of pipe, $D$ & 0.9 & $\mathrm{~m}$ \\
\hline Wall thickness of pipe, $e$ & 0.017 & $\mathrm{~m}$ \\
\hline Density of water, $\rho_{w}$ & $10^{3}$ & $\mathrm{~kg} / \mathrm{m}^{3}$ \\
\hline Dynamic viscosity of water, $\mu_{w}$ & $10^{-3}$ & $\mathrm{Pa.s}$ \\
\hline Wave speed of water, $a_{w}$ & 1037.57 & $\mathrm{~m} / \mathrm{s}$ \\
\hline Elastic modulus of water, $K_{w}$ & $2.19 .10^{9}$ & $\mathrm{GPa}$ or $\mathrm{N} / \mathrm{m}^{2}$ \\
\hline Density of slurry, $\rho_{s}$ & 1600 & $\mathrm{~kg} / \mathrm{m}^{3}$ \\
\hline Dynamic viscosity of slurry, $\mu_{s}$ & 0.0102 & $\mathrm{Pa.s}$ \\
\hline Wave speed of slurry, $a_{s}$ & 971.34 & $\mathrm{~m} / \mathrm{s}$ \\
\hline Elastic modulus of phosphate, $K_{f}$ & $8.10^{10}$ & $\mathrm{GPa}$ or $\mathrm{N} / \mathrm{m}^{2}$ \\
\hline Young modulus, $E$ & $112.10^{9}$ & $\mathrm{~N} / \mathrm{m}^{2}$ \\
\hline Final time, $T_{\max }$ & 252 & $\mathrm{~s}$ \\
\hline Concentration of solid phase, $C_{s}$ & 0.4 & dimensionless \\
\hline
\end{tabular}

Six targeted flashed images are illustrated in Fig. 8 to simulate the unsteady-state of three-fluid flow. This head $H_{0}$

defined by equality (50) is shown in picture $8 \mathrm{a}$. This initial head $H_{0}$ is a discontinuous function due to water's density being less than liquid slurry's. This comparison is justified by using equality (21). It is concluded that the friction coefficient $f_{w}$ is less than the slurry one $f_{s}$, showing that the hydraulicgrade-line elevation for slurry is under water.

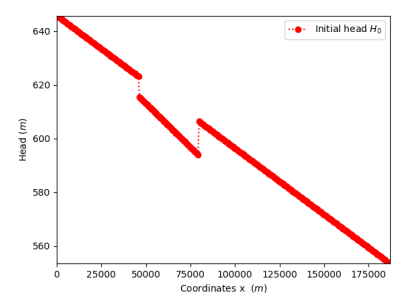

(a) Initial head $H_{0}(x)$.

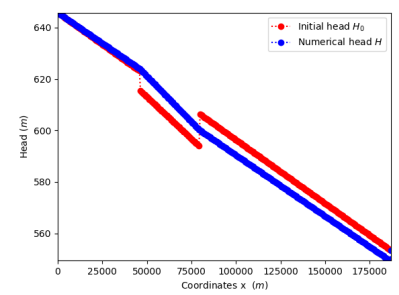

(c) Head $H(t, x)$ at $T_{\max }$ compared to $H_{0}$.

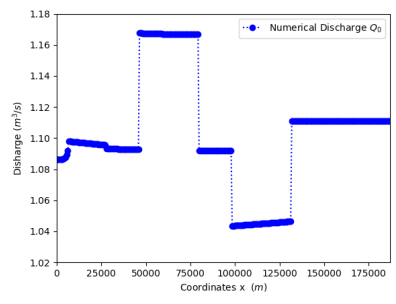

(e) Discharge $Q(t, x)$ at instant 84.

Fig. 8: Evolution of head-discharge over space in unsteadystate.

The interpretation of the simulation defined in pictures $8 \mathrm{~b}$ and $8 \mathrm{c}$ are given as follows. The flow is shared into two parts. The first one $L_{1}$ is constituted with left water and slurry. The mixture of these two liquids forms one having an elevation which is close to slurry one. This liquid goes to the left side and reflects. The second one $L_{2}$ is constituted with slurry and right water. The fluid's particles generated by this mixture move over the pipeline. At time 84, the interaction between liquids $L_{1}$ and $L_{2}$ give fluid's particles that are ready to leave the right side. Insisting here that instant 84 is given by $T_{\max }$ per 3 , and it is valid in the rest of the paper. This instant means occurring the move forward of slurry from middle section to the next one. Showing its transport is important in modeling framework. The picture 8c shows that these particles exit the pipeline at instant $T_{\max }$. Only one mixture of liquids $L_{1}$ and $L_{2}$ dominates the flow generating one fluid at the final time.

Now, we give how the discharge varies in pictures $8 \mathrm{~d}, 8 \mathrm{e}$ and 8 f. Setting the initial discharge $Q_{0}$ to value $\frac{10}{9}$, i.e. $Q_{0}=$ 
$\frac{10}{9}$. This discharge $Q_{0}$ defined by equality (49) is shown in picture $8 \mathrm{~d}$, meaning that is constant at initial time in terms of position's pipeline. In the beginning of this simulation, the discharge is ever constant in the second region. It follows that at instant 84, the moving slurry's discharge is less than the water one (see picture $8 \mathrm{e}$ ). The model converges to a piecewise function presented in picture 8f. It is allowing to generate the same discharge in the first and last regions, and greater one in the middle region.

Consequently, these researches given by pictures 6 and 8 focused on predicting pressure drop and discharge in cement slurry flow in circular pipe. Results showed that regime unsteady-state determine only one state in the final stage. This slurry was produced over time with mixing phosphate and water. When convergence time occurs, the final state shows that the head is homogeneous. Since single-phase fluid is pipelined at large distances, slurry particles are concentrated on the bottom of the pipeline. At instant $T_{\max }$, this mixture exit from the pipe. Regarding discharge $Q$, it is the same one at inlet and outlet sections, even it is a discontinuous function in space.

\section{COUPLING MODELS}

In this section, we keep the same modeling introduced previously in section VI as shown in Fig. 9. We are interested in transport of three-fluid through a pipeline in unsteadystate. Here, three artificial conditions are imposed at each part of sections. In this model, the fluid flow in each section does not impact the other one. It means that the flow is not transmitted from one section to another one. There is any influence between slurry and water. To develop this model, equations (10) and (12) must be solved together in each section of the pipeline independently on other. These equations are solved using the method of characteristics. Noticing that this model does not reflect the physical reality. However, it is built in order to be converged at final time to the steady-state.

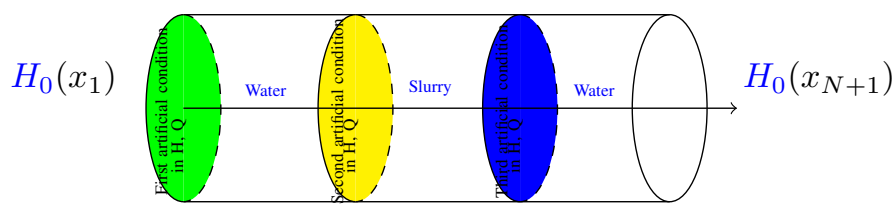

Fig. 9: Schematics of water-slurry-water flow in a circular pipe with artificial conditions.

The initial discharge $Q_{0}^{i}$ is defined in terms of two constants $Q_{0}$ and $Q_{1}$ :

$$
Q_{0}^{i}(x)=\left\{\begin{array}{l}
Q_{0}, x \in\left[x_{1}, x_{N_{1}+1}\right] \\
\left.\left.Q_{1}, x \in\right] x_{N_{1}+1}, x_{N_{2}+1}\right] \\
\left.\left.Q_{0}, x \in\right] x_{N_{2}+1}, x_{N+1}\right]
\end{array}\right.
$$

and the initial head $H_{0}^{i}$ is given by:

$$
H_{0}^{i}(x)=\left\{\begin{array}{l}
H\left(x_{1}\right), x \in\left[x_{1}, x_{N_{1}+1}\right] \\
\left.\left.H\left(x_{1}\right)-\frac{f_{s} Q_{1}\left|Q_{1}\right|}{2 g D A^{2}}\left(x_{N_{1}+1}-x_{1}\right), x \in\right] x_{N_{1}+1}, x_{N_{2}+1}\right] \\
\left.\left.H\left(x_{1}\right)-\frac{f_{w} Q_{0}\left|Q_{0}\right|}{2 g D A^{2}}\left(x_{N_{2}+1}-x_{1}\right), x \in\right] x_{N_{2}+1}, x_{N+1}\right]
\end{array}\right.
$$

We will apply the Dirichlet boundary conditions for the head $H$ at the upstream boundary of each section. These upstream boundaries are given by quantities $H\left(x_{1}\right), H\left(x_{N_{1}+1}\right)$, and $H\left(x_{N_{2}+1}\right)$ which are defined respectively by:

$$
\begin{gathered}
H\left(x_{1}\right)=H_{0}^{i}\left(x_{1}\right) . \\
H\left(x_{N_{1}+1}\right)=H\left(x_{1}\right)-\frac{f_{s} Q_{1}\left|Q_{1}\right|}{2 g D A^{2}}\left(x_{N_{1}+1}-x_{1}\right) . \\
H\left(x_{N_{2}+1}\right)=H\left(x_{1}\right)-\frac{f_{w} Q_{1}\left|Q_{1}\right|}{2 g D A^{2}}\left(x_{N_{2}+1}-x_{1}\right) .
\end{gathered}
$$

The head at the downstream boundary is defined by the Neumann condition at each parts of section. In what to follow, for the discharge $Q$, we impose the Neumann condition at the upstream boundary. On the contrary, the Dirichlet condition is adjusted at the downstream boundary.

The variation of head-discharge is shown in Fig. 10 that is shared into two pictures, given below:
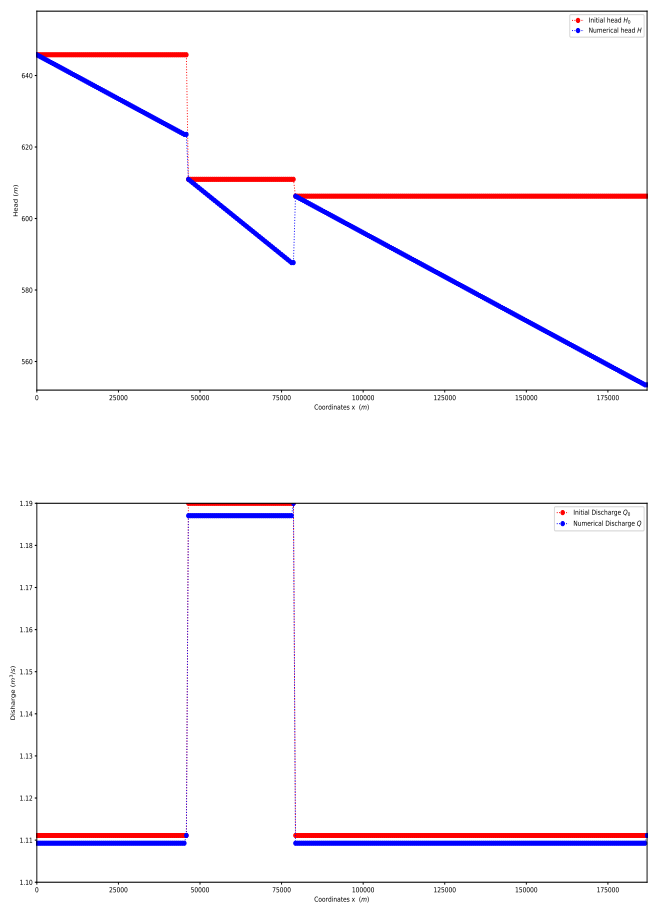

Fig. 10: Coupling models and evolution of head-discharge over space in unsteady-state. 
The initial head $H_{0}$ defined by equality (55) is shown in the top picture. This head is a piecewise constant function, which is equal to $H\left(x_{1}\right)$ over the first region, to $H\left(x_{1}\right)-$ $\frac{f_{s} Q_{1}\left|Q_{1}\right|}{2 g D A^{2}}\left(x_{N_{1}+1}-x_{1}\right)$ between positions $x_{N_{1}+1}$ and $x_{N_{2}+1}$ and to $H\left(x_{1}\right)-\frac{f_{w} Q_{0}\left|Q_{0}\right|}{2 g D A^{2}}\left(x_{N_{2}+1}-x_{1}\right)$ over the last region. The discharge is initialized according to equality (54), that is shown in the bottom picture, and its evolution until instant $T_{\max }$ is presented in the same picture. The head converges to a piecewise affine function when time goes to the final instant $T_{\max }$. This elevation of the three-fluid model is not the same, showing that the fluid is not homogeneous due to utilization of three different artificial conditions. Noticing that we showed in section $\mathrm{V}$ that each component head converges to affine function with a decreasing slope. In the same case the discharge remains the same. The same reasoning is available for head-discharge constituting each piece, justifying this result. It is concluded that the unsteady flow converges to the steady-state, achieving the validation of this model.

\section{CONCLUSION}

The slurry transportation has been mainly studied and it is shown that the flow is stable in presence of water. In summary, we have applied the method of characteristics for compressible multi-fluid flow which is described by hyperbolic systems. We have tested over this paper the ability of the code to conserve the pipeline in steady-state. We have shown also the results that regime unsteady-state determine the solution in different time durations. Additionally, the numerical results were analyzed based on various flow conditions. In the future, we would like to extend the numerical code for the simulation to higher dimensions, and generalize it particularly to threedimensional problems fairly easily. On the other hand, even the method of characteristics is efficient compared to the finite volume method [27], and it is capable of the advantages of high accuracy. Moreover, numerical tests will be performed by comparing results of these two numerical methods. To analyze two-phase flow under the influence of various floating particles, it is necessary to develop a new model which investigates the mechanism of their interaction. A few recent works have proposed this study [28], [29], [30]-[31]. On others words, establishing the multiphase model with considering solid-liquid two-phase coupling, as mentioned in [32]. It is observed that computational fluid dynamics (CFD) modeling gives fairly good results to simulate the slurry flow in pipeline [33], [34]. It is interesting to use CFD calculations by using Eulerian model and compare it with the developed one.

\section{ACKNOWLEDGMENT}

I would like to thank the anonymous reviewers for their helpful comments and valuable suggestions to improve quality of this article. This work was supported by the research federation Math-STIC, University Paris 13.

\section{REFERENCES}

[1] A. Mackenzie, M. T. SticklandW and M. Dempster, "Development of a Combined Euler-Euler Euler-Lagrange Slurry Model," OpenFOAM®, pp. 77-91, 2019.
[2] M. H. Kasbaoui, D. L. Koch and O. Desjardins, "Clustering in Euler-Euler and Euler-Lagrange simulations of unbounded homogeneous particle-laden shear,' Journal of Fluid Mechanics, vol. 859, pp. 174-203, 2019.

[3] S. A. Miedema, "A head loss model for homogeneous slurry transport for medium sized particles," Journal of Hydrology and Hydromechanics, vol. 63, no. 1, pp. 1-12, 2015.

[4] F. Benkhaldoun, I. Elmahi and M. Sea1, "Well-balanced finite volume schemes for pollutant transport by shallow water equations on unstructured meshes," Journal of computational physics, vol. 226, no. 1, pp. 180-203, 2007.

[5] T. Chakkour, "Simulations numériques des tubes avec contraction brusque sur openfoam," Thermodynamique des interfaces et mécanique des fluides, vol. 17, no. 1, 2017.

[6] M. Ishii and K. Mishima, "Two-fluid model and hydrodynamic constitutive relations," Nuclear Engineering and design, vol. 82, no. 2-3, pp. 107-126, 1984.

[7] J. Wang, S. Wang, T. Zhang and F. Battaglia, "Mathematical and experimental investigation on pressure drop of heterogeneous ice slurry flow in horizontal pipes," International Journal of Heat and Mass Transfer, vol. 108, pp. 2381-2392, 2017.

[8] G. Shou, Slurry pipeline system: Simulation and validation

[9] J. Rusconia, A. Lakhouajab, and M. Kopuzc, "The Design and Engineering of the $187 \mathrm{~km}$ Khouribga to Jorf Lasfar Phosphate Slurry Pipeline," Procedia Engineering, vol. 138, pp. 142-150, 2016.

[10] B. Geissler, L. Hermann, M. C. Mew and G. Steiner, "Striving toward a circular economy for phosphorus: the role of phosphate rock mining," Minerals, vol. 8, no. 9, pp. 395-417, 2018.

[11] S. Yao, Y. Li, W. Wang, G. Song, Z. Shi, X. Wang, and S. Liu, "Investigation of hydrate slurry flow behaviors in deep-sea pipes with different inclination angles," Oil \& Gas Science and Technology-Revue d'IFP Energies nouvelles, vol. 74, pp. 48-60, 2019.

[12] B. Shi, Y. Liu, L. Ding, X. Lv, J. Gong, "New Simulator for Gas-Hydrate Slurry Stratified Flow Based on the Hydrate Kinetic Growth Model," Journal of Energy Resources Technology, vol. 141, no. 1, 2019.

[13] D. J. Wood and T. Y. Kao, "Unsteady flow of solid-liquid suspensions," Journal of the Engineering Mechanics Division, vol. 92, no. 6, pp. 117134, 1966.

[14] W. Bechteler and G. Vogel, "Pressure wave velocity in slurry pipelines," Proceedings of Hydrotransport, vol. 8, 1982.

[15] ARD. Thorley and L. Y. Hwang, "Effects of rapid change in flowrate of solid-liquid mixtures," Proceedings of Hydrotransport 6th Conference, $U K$, pp. 229-242, 1979.

[16] E. B. Wylie, V. L. Streeter and L. Suo, "Fluid transients in systems," Prentice Hall Englewood Cliffs, vol. 1, 1993.

[17] E. B. Wylie and V. L. Streeter, "Fluid transients," New York, McGrawHill International Book Co., 1978.

[18] M. Grozdek, R. Khodabandeh and P. Lundqvist, "Experimental investigation of ice slurry flow pressure drop in horizontal tubes," Experimental Thermal and Fluid Science, vol. 33, no. 2, pp. 357-370, 2009.

[19] J. T. Park, R. J. Mannheimer, T. A. Grimley, and T. B. Morrow, "Pipe flow measurements of a transparent non-newtonian slurry," Journal of fluids engineering, vol. 111, no. 3, pp. 331-336, 1989.

[20] R. W. Hanks and B. H. Dadia, "Theoretical analysis of the turbulent flow of non-newtonian slurries in pipes," AIChE Journal, 1971.

[21] R. Darby and J. Melson, "How to predict the friction factor for flow of bingham plastics," Chemical Engineering, vol. 88, no. 26, pp. 59-61, 1981.

[22] R. Darby, R. Mun and D. V. Boger, "Predict friction loss in slurry pipes," Chemical Engineering, vol. 99, no. 9, pp. 116-119, 1992.

[23] E. Buckingham, "On plastic flow through capillary tubes," ProceedingsAmerican society for testing and materials, pp. 1154-1156, 1921.

[24] S. W. Churchill, "Friction-factor equation spans all fluid-flow regimes," Chemical engineering, vol. 84, no. 24, pp. 91-92, 1977.

[25] R. J. Fennema and M. H. Chaudhry, "Explicit numerical schemes for unsteady free-surface flows with shocks," Water Resources Research, vol. 22, no. 13, pp. 1923-1930, 1986.

[26] M. B. Holloway and M. H. Chaudhry, "Stability and accuracy of waterhammer analysis," Advances in Water Resources, vol. 8, 1985.

[27] R. Liu, D. Wang, X. Zhang, W. Li, and B. Yu, "Comparison Study on the Performances of Finite Volume Method and Finite Difference Method," Journal of Applied Mathematics, vol. 2013, 2013.

[28] Y. H. Song, E. H. Lee and J. H. Lee, "Functional relationship between soil slurry transfer and deposition in urban sewer conduits," Water, vol. 10 , no. 7 , pp. $825-841,2018$. 
[29] S. Akhtar, H. Ali and C. W. Park, "Thermo-Fluidic Characteristics of Two-Phase Ice Slurry Flows Based on Comparative Numerical Methods," Processes, vol. 7, no. 12, pp. 898-914, 2019.

[30] A. Amani, N. Balcázar, A. Naseri, J. Rigola, "A numerical approach for non-Newtonian two-phase flows using a conservative level-set method," Chemical Engineering Journal, vol. 385, pp. 123896-123916, 2020.

[31] T. Chakkour, "Application of two-dimensional finite volume method to protoplanetary disks," Astronomy Letters, under review, 2020

[32] X. J. Shi and P. Zhang, "Two-phase flow and heat transfer characteristics of tetra-n-butyl ammonium bromide clathrate hydrate slurry in horizontal $90^{\circ}$ elbow pipe and U-pipe," International Journal of Heat and Mass Transfer, vol. 97, pp. 364-378, 2016.

[33] O. Parkash, A. Kumar, B. S. Sikarwar, "CFD Modeling of Commercial Slurry Flow Through Horizontal Pipeline," Advances in Interdisciplinary Engineering, pp. 153-162, 2019.

[34] A. Kumar, "CFD Modeling for Slurry Flow Through Bends and Straight Pipe Line," CAD/CAM, Robotics and Factories of the Future, pp. 615623, 2016.

[35] C. Tao, B. G. Kutchko, E. Rosenbaum and M. Massoudi, "A Review of Rheological Modeling of Cement Slurry in Oil Well Applications," Energies, vol. 13, no. 3, pp. 570-622, 2019.

[36] N. Kumar, M. K. Gopaliya, D. R. Kaushal, "Experimental investigations and CFD modeling for flow of highly concentrated iron ore slurry through horizontal pipeline," Particulate Science and Technology, vol. 37, no. 2, pp. 232-250, 2019.

[37] T. Chakkour, "Some notes about the continuous-in-time financial model," Abstract and Applied Analysis, vol.2017, 2017, Hindawi.

[38] T. Chakkour, "Inverse problem stability of a continuous-in-time financial model," Acta Mathematica Scientia, vol. 39, no. 5, pp. 1423-1439, 2019.

[39] G.Camera-Roda, V.Augugliaro, A. G. Cardillo, V.Loddo, L. Palmisano, F.Parrino, F.Santarelli, "A reaction engineering approach to kinetic analysis of photocatalytic reactions in slurry systems," Catalysis Today, vol. 259, pp. 87-96, 2016.

[40] N. Kumar, M. K. Gopaliya and D. R. Kaushal, "Experimental investigations and CFD modeling for flow of highly concentrated iron ore slurry through horizontal pipeline," Particulate Science and Technology, vol. 37, no. 2, pp. 232-250, 2019.

[41] J. R. Januário and C. B. Maia, "CFD-DEM simulation to predict the critical velocity of slurry flows," Journal of Applied Fluid Mechanics, vol. 13, no. 1, pp. 161-168, 2020.

[42] G. M. Oliveira and A. T. Franco, "Mathematical Model for Viscoplastic Fluid Hammer," Journal of Fluids Engineering, vol. 138, no. 1, 2016.

[43] N. Ijaz, A. Zeeshan, M. M. Bhatti, R. Ellahi, "Analytical study on liquid-solid particles interaction in the presence of heat and mass transfer through a wavy channel," Journal of Molecular Liquids, vol. 250, pp. 80-87, 2018. 